\title{
Ground-based Fabry-Pérot interferometry of the terrestrial nightglow with a bare charge-coupled device: remote field site deployment
}

\author{
Rick Niciejewski, MEMBER SPIE \\ Timothy L. Killeen \\ Matthew Turnbull \\ University of Michigan \\ College of Engineering \\ Space Physics Research Laboratory \\ Department of Atmospheric, Oceanic, and \\ Space Sciences \\ Ann Arbor, Michigan 48109-2143
}

\begin{abstract}
The application of Fabry-Pérot interferometers (FPIs) to the study of upper atmosphere thermodynamics has largely been restricted by the very low light levels in the terrestrial airglow as well as the limited range in wavelength of photomultiplier tube (PMT) technology. During the past decade, the development of the scientific grade charge-coupled device (CCD) has progressed to the stage in which this detector has become the logical replacement for the PMT. Small fast microcomputers have made it possible to "upgrade" our remote field sites with bare CCDs and not only retain the previous capabilities of the existing FPls but expand the data coverage in both temporal and wavelength domains. The problems encountered and the solutions applied to the deployment of a bare CCD, with data acquisition and image reduction techniques, are discussed. Sample geophysical data determined from the FPI fringe profiles are shown for our stations at Peach Mountain, Michigan, and Watson Lake, Yukon Territory.
\end{abstract}

Subject terms: magnetospheric imagery; atmospheric remote sensing: FabryPérot interferometry; atmospheric remote sensing.

Optical Engineering 33(2), 457-465 (February 1994).

\section{Introduction}

One goal within the National Science Foundation's Coupling. Energetics, and Dynamics of Atmospheric Regions Program (NSF-CEDAR) is to develop and deploy state-of-the-art instrumentation at field sites scattered across the globe that will perform unattended automatic data acquisition of terrestrial airglow and auroral emissions. These data sets provide a means to characterize the weather at high-altitude regions of the atmosphere: the mesosphere, the lower and upper thermosphere, and the exosphere. Simple optical experiments controlled by small data storage instruments are one technique employed by aeronomers. In particular, Fabry-Pérot interferometers (FPIs), suitably outfitted, are capable of monitoring neutral and ion temperatures, neutral horizontal and vertical winds, ion drifts, and composition measurements from stable environmentally controlled platforms. Other instruments used by experimental optical aeronomers are diffraction grating spectrometers or spectrographs, spectrophotometers, all-sky imaging cameras, and Fourier transform spectrometers. The common connecting thread of all these techniques is the desire to perform very low light level ex-

Paper M1-039 received July 1, 1993; revised manuscript received Aug. 25, 1993 accepled for publication Aug. 30, 1993. This paper is a revision of a paper presented at the SPIE conference on Instrumentition for Planctary and Terrestrial Atmospheric Remote Sinsing. July 1992, San Diego. Calil. The puper prevented there appears (unrefereed) in SPIE Proceedings Vol. 1745.

(c) 1994 Society of Photo-Optical Instrumentation Enginters. 01091-3286/94/\$6. (60. periments on temporally varying phenomena to extract geophysical parameters with the lowest possible error bars.

We describe the current technique used by a group of aeronomers at the University of Michigan to perform FabryPérot interferometry at remote field sites. These locations are typically difficult to reach, have little if any on-site support, are in harsh environments, and usually are isolated from the principal investigators for large parts of the observing season. The FPI ${ }^{1,2}$ measures the line profile of an emission originating from a long-lived state of either atomic oxygen (ground or singly ionized state) or hydroxyl. The excited state has a lifetime sufficient for it to reach local thermodynamic equilibrium and, hence, provides a measure of the local temperature. Shifts in the peak location provide an indication of the line-of-sight velocity, which may easily be converted into either a horizontal (meridional and/or zonal) or a vertical wind component. The key measurement of these FPI airglow experiments is the 2-D fringe profile-intensity versus phase difference. We discuss the method employed to image the fringe profile and to extract the fringe profile from the image, as well as the difficult task of deploying a bare CCD as the sensor for an FPI airglow experiment.

The FPI instruments deployed are all located in the northern hemisphere: Thule Air Base, Greenland; Søndre Strømfjord, Greenland; Watson Lake, Yukon Territory, Canada; and Peach Mountain, Michigan. The Greenland sites currently use an image plane detector ${ }^{3}$ to gather light, while 
the latter two employ a bare CCD as a detector. As we learn to deal with cryogen coolant, the Sondre Stromfjord facility will be upgraded with a bare CCD. In addition, an all-sky imaging experiment was redeployed at Peach Mountain with a new upgrialed bare CCD detector during the fall of 1992.

\section{Experiment}

The absolute radiative quantity measured, known as the integrated intensity or surface brightness, is usually assumed to be isotropic, and within the aeronomical community is scaled by the absolute unit, ${ }^{+}$the rayleigh $(R)$, where $I R$ is equivalent to $10^{\circ}$ photons $\mathrm{cm}^{-2}$ (column) $\mathrm{s}^{-1}$. The absolute emission rates for the features observed by the FPI are in the neighborhood of 10 to $100 \mathrm{R}$, in which $1 \mathrm{kR}$ is typical of the limit of naked eye visibility. Hence, the FPI airglow experiment must be capable of measuring very low light levels with minimal contribution from inherent noise sources.

An example of our typical FPI airglow experiment is given in Meriwether et al. ${ }^{5}$ All stations have basically the same physical layout, but use one of two differently styled detectors. The image plane detectors employed in Greenland consist of only 12 channels, while the CCDs at Peach Mountain and in the Yukon have thousands of individual pixels that may be ordered into $\sim 100$ channels. Consequently, with appropriate optical elements, a CCD-based FPI may be designed to image several fringes simultaneously, an inherent multiplex advantage over the Greenland image plane detector (IPD)-based FPI

\section{Charge-Coupled Device Selection}

The primary requirements for the choice of a bare CCD for our FPI airglow experiments are low inherent thermal noise and low readout noise. The portion of the CCD used in the FPI airglow experiment is a circular area closely aligned with the center of the detector and, hence, defects around the edges of the CCD are not important for our photometric work. This latter point permits some reduction in sensor cost, because a perfect detector is not required for the experiment. Our experiments are conducted with visible- or near-infrared radiation. therefore, front-illuminated devices have satisfactory quantum efficiency for our requirements. In the last several years, our group has experimented with four different CCDs for use within FPI and all-sky imaging experiments: (1) a Fairchild CCD222 device, (2) a Thomson TH7882 CDA device. (3) a Ford/Photometrics PM5 12 device, and (4) a Kodak KAF-1400 device. The numerical order matches our chronological purchasing and, coincidentally, cost (the more recent the purchase, the greater the cost). Note that (2) and (3) are no longer in production. Device (1) was initially used with a lab bench FPI before installation in an all-sky imaging experiment. We have recently replaced the Fairchild chip with another Kodak device and have fielded this upgrade. Devices (2). (3), and (4) have been fielded in Ann Arbor, Peach Mountain, and Watson Lake, though we will soon move device (3) to Søndre Stromfjord, returning (2) to active use. Table 1 describes the devices according to manufacturer specifications. We have performed some checks on these specifications, though a report is usually issued on receipt of each camera system.

The Fairchild CCD is a fairly inexpensive design that has been used in several other aeronomical applications: for ex-
Table 1 Specifications of bare CCD devices employed for FPI studies. The primary characteristic that determines the usefulness of the CCD is the noise level. In use, the Thomson chip is binned $3 \times 3$, the PM512 chip is binned $4 \times 4$, and the Kodak chip is binned $8 \times 8$. the dark noise component of the This on-chip binning increases the dark noise component of the super pixel by the square root of the number of component pixels, but the readout noise associated with the

\begin{tabular}{lccll}
\multicolumn{1}{c}{ Device } & $\begin{array}{c}\text { Number of } \\
\text { pixels }\end{array}$ & $\begin{array}{c}\text { Pixel } \\
\text { size }(\mu \mathrm{m})\end{array}$ & $\begin{array}{c}\text { Readout noise } \\
(\mathrm{e}-\mathrm{RMS})\end{array}$ & $\begin{array}{c}\text { Dark noise } \\
(\mathrm{e}-\text { sec pixel) }\end{array}$ \\
& & & & \\
Fairchild CCD222 & $488 \times 380$ & $12 \times 18$ & 60 & $18000 @ 24^{\circ} \mathrm{C}$ \\
Thomson TH7882 CDA & $384 \times 576$ & $23 \times 23$ & $11.5 \mathrm{w} / \mathrm{bias}$ & $4.1 \mathrm{e} / \mathrm{min} @-120^{\circ} \mathrm{C}$ \\
PM512 & $516 \times 516$ & $20 \times 20$ & 8 & $6.0 @-45^{\circ} \mathrm{C}$ \\
Kodak KAF1400 & $1317 \times 1035$ & $6.8 \times 6.8$ & 11.2 & $0.003 @-42^{\circ} \mathrm{C}$
\end{tabular}

ample, see Torr et al. ${ }^{6}$ The system was purchased in a singledetector CAM/CCD5000 design in which the CCD resided in the CAM5000 sense head with a Peltier-effect cooler capable of reducing the $\mathrm{CCD}$ die temperature by $20^{\circ} \mathrm{C}$ below ambient. The control unit, a CCU5000 camera subsystem, provided output in RS170A television format, implying a 1.4-V peak-to-peak $75-\Omega$ source impedance, composite analog video output. This translates into a 525 -line, $30-\mathrm{Hz}$ frame rate, which is acceptable for bright signals but useless for low light level sources. Modifications to the hardware were made in our lab to (1) remove an IR filter from the sense head enclosure to extend the wavelength sensitivity of the device, (2) permit time exposures with integrations ranging up to several minutes. and (3) further cool the sense head by placing it within a Products for Research (PfR) thermoelectric cooler. The latter modification allowed the CCD to be cooled to about $-35^{\circ} \mathrm{C}$, which reduced a very large thermal noise component. The final design was fielded at Thule Air Base within an all-sky imager devoted to monitoring auroral and airglow patches during the long polar winter nights. Data are stored in analog form on VHS videotape, though a frame grabber board within the data control system permits digitization of either real-time analog signals or previously recorded images.

Initial dark tests with the Fairchild CCD indicated that without the PfR cooler, the CCD was unacceptable for low light level studies. The detector saturated in $20 \mathrm{~s}$, as indicated by the average 8 -bit digital pixel value. Further test results with the CAM5000 placed inside the PfR cooler permitted observations of several minutes. However, airglow observations were still difficult to make, though auroral measurements at Thule Air Base were possible, proving our conceptual design. ${ }^{?}$

The Thomson CCD system was purchased from Photometrics, Ltd., with a liquid nitrogen cooling requirement. The use of cryogen permits a drastic reduction in the operating die temperature of the $\mathrm{CCD}$, with a consequent reduction in thermal noise. The system was ordered with a CC200 camera electronics unit, a CC200 camera controller, and a sidelooking $\mathrm{CH} 210$ cryogenic camera head. Note that these components, as described, are no longer available trom Photometrics, including the Thomson TH7882 CDA sensor. The entire experiment was initially controlled via a GPIB interface with a Micro Vax II controller, though later, an IBM AT clone supplanted the larger computer. Our first successful 


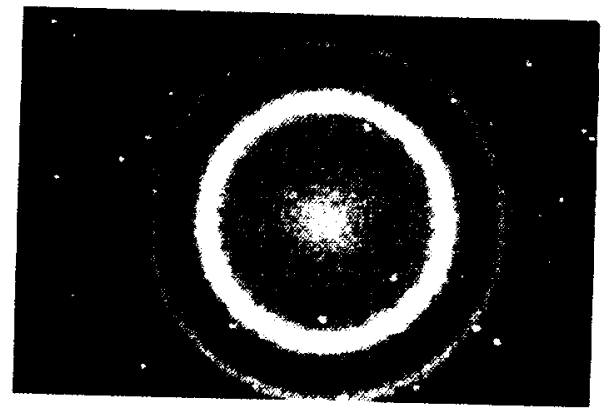

(a)

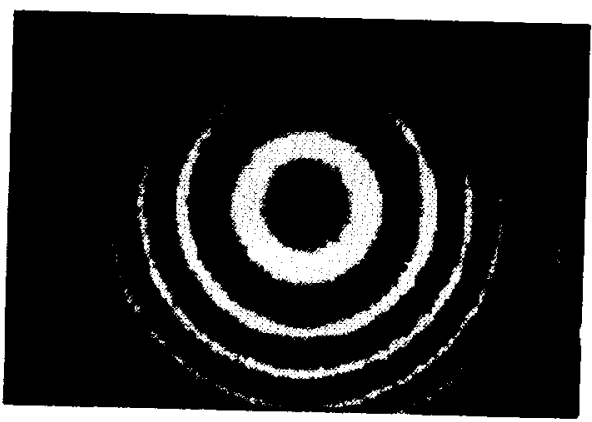

(c)

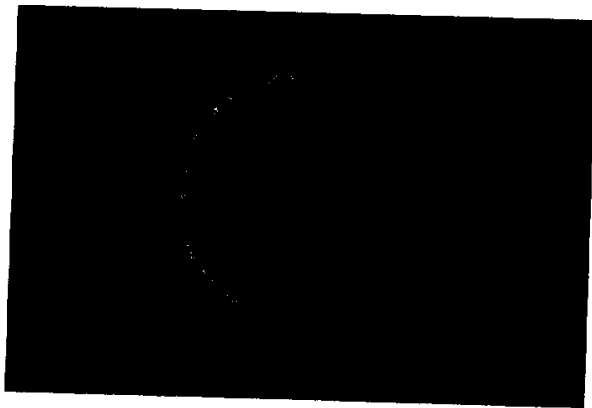

(b)

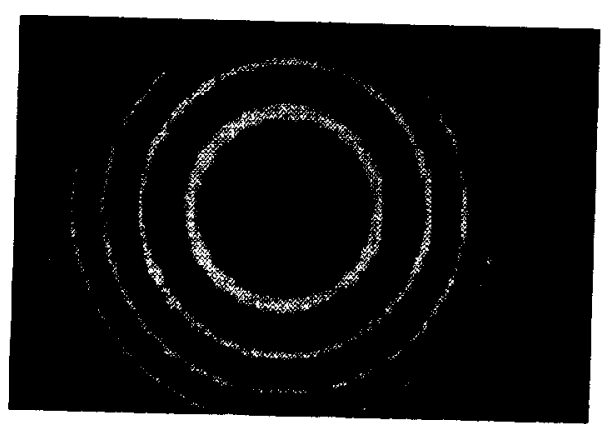

(d)

Fig. 1 FPI images acquired by the Peach Mountain [(a) and (b)] and the Watson Lake [(c) and (d)]
systems: (a) a 5-min integration through a filter isolating systems: (a) a 5-min integration through a filter isolating emission from the lower thermosphere OI (c) a 1-min integration a 5-min integration through a filter isolating a mesospheric hydroxyl emission, (c) a 1-min integration of $\mathrm{Ol}(6300-\AA)$ emission, and (d) a HeNe image.

airglow FPI observations were performed with this system at a lab in Ann Arbor, Michigan. Beginning in September 1992, this system began remote unattended operation at a field site at a dark location on Peach Mountain, Michigan. Figure 1 contains two images taken with this system: (a) shows an image of an OI $(5577-\AA)$ fringe, while (b) shows an image of the OH-Meinel $(7,3) \mathrm{P}_{1}(3)$ line at $8920 \AA$.

The PM512 CCD system was also purchased from Photometrics, Ltd., with components similar to the Thomson system, though the $\mathrm{CH} 210$ dewar was of the bottom-looking variant. This required the addition of another small mirror in the optical path to bend the light beam onto the sensor, rather than placing the dewar on its side (LN2 would have dribbled out). The original set of software control routines designed to operate the Thomson-based system required some revision to handle the different pixel arrangement of the PM512. Otherwise, the PM512 and the Thomson systems were identical. This system has been evaluated for long-term unattended operation at a field site on Peach Mountain, Michigan, and will soon be transferred to an aeronomical observatory in Søndre Strømfjord, Greenland. Geophysical data were acquired in support of a CEDAR-sponsored mid-latitude, mesosphere, and lower thermosphere thermodynamic project, as well as ground truth support for the orbiting UARS mission.

The Watson Lake, Yukon, facility was deployed in November 1991 as a remote upper thermosphere monitoring station. The FPI was an old design originally deployed in Alaska and then moved to Calgary, Alberta, in 1980. The detector used in Calgary was an IPD device that acquired lower thermosphere measurements ${ }^{5}$ during the early 1980s. Equipment failure and a lack of funding precluded any further experimental measurements until 1991. The optical hardware was returned to Michigan and a bare CCD-based system was purchased from Photometrics, Ltd. This time, a Kodak KAF $1400 \mathrm{CCD}$ was chosen as the sensor. The rest of the system comprised a $\mathrm{CH} 250$ camera head, an LC200 liquid circulation unit, and an IBM AT interface card. This system differed markedly from the older Photometrics versions in that it was liquid cooled rather than cryogen cooled, thus making the detector head more compact. The Kodak CCD operates solely in the multipinned phase (MPP) mode ${ }^{8}$ and. thus, the expected enhanced thermal noise contribution from operating at $-40^{\circ} \mathrm{C}$ is much reduced. Figures $\mathrm{l}(\mathrm{c})$ and 1 (d) display fringes acquired by this system: I(c) shows an image of $\mathrm{OI}(6300-\AA)$ fringes, while $1(\mathrm{~d})$ shows an image of HeNe laser fringes

The chronological history of our attempts at using and upgrading very low light level hardware shows that we have been very successful. Three FPIs use a bare CCD device and an all-sky imager has been outfitted with another Kodak device. The sensitivity range of the $C C D$ is such that near-IR radiation may be easily observed, adding a new dimension to our experiments-hydroxyl emissions become brighter as one moves from the visible to the near IR, allowing groundbased remote observation of mesospheric altitudes. As funds become available, remaining FPIs and diffraction grating spectrometers will be upgraded with the versatile $C C D$.

\section{Fabry-Pérot Interferometry with a Bare Charge-Coupled Device.}

\subsection{Fitting a Circular Fringe onto a Square Matrix} Initial observations of frequency-stabilized HeNe laser fringe 


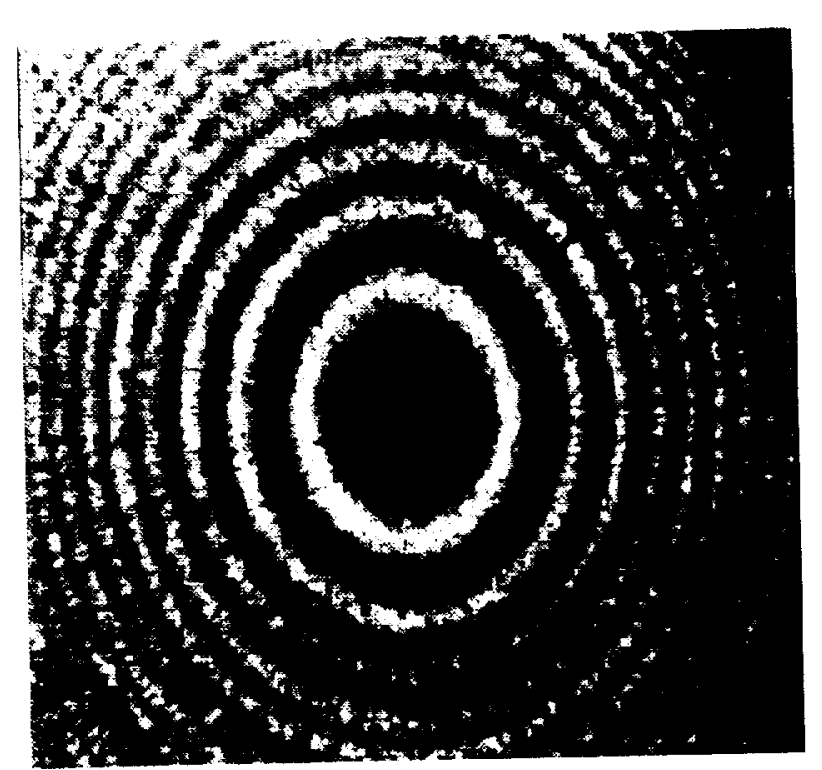

(a)

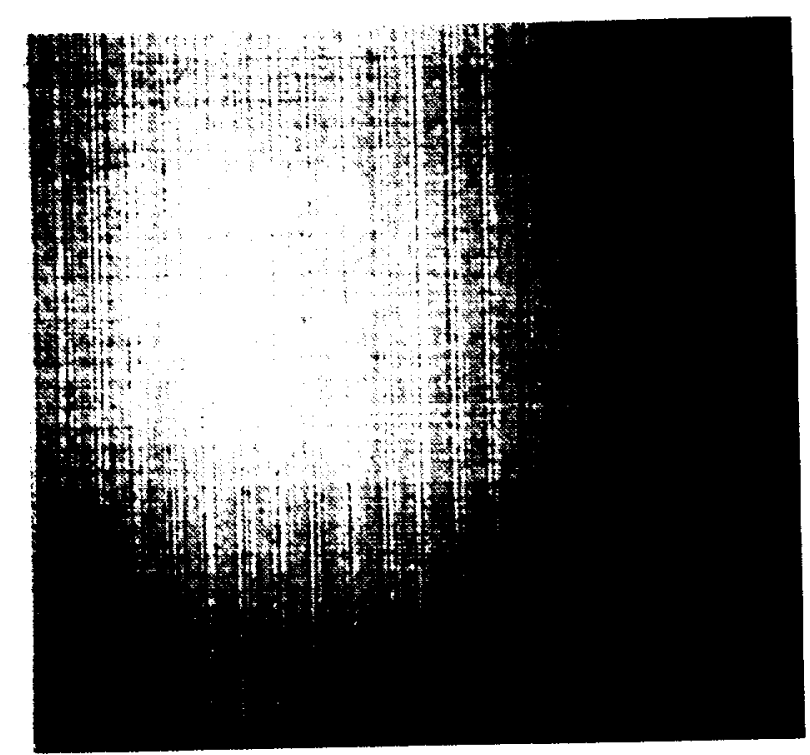

(c)

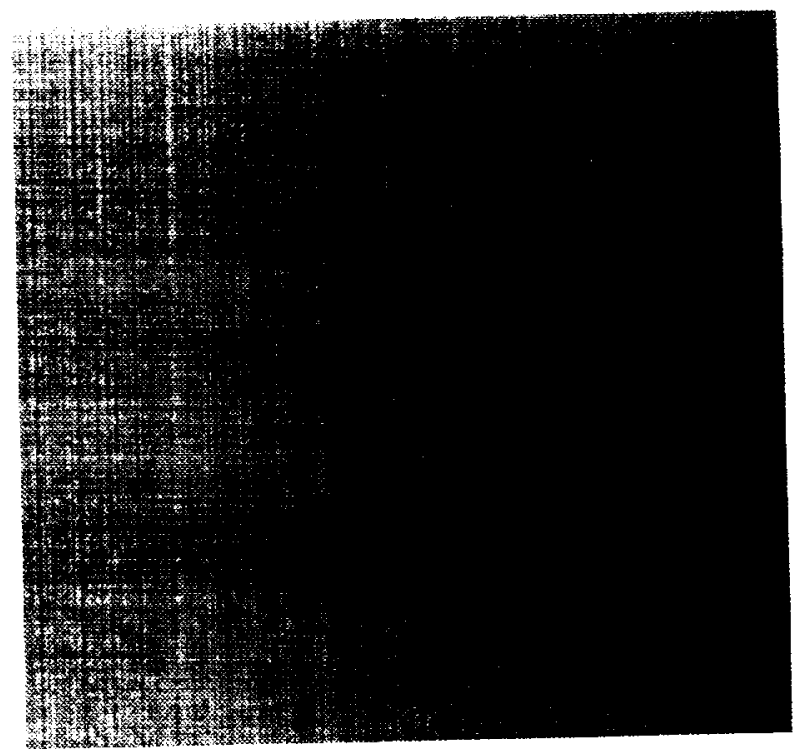

(b)

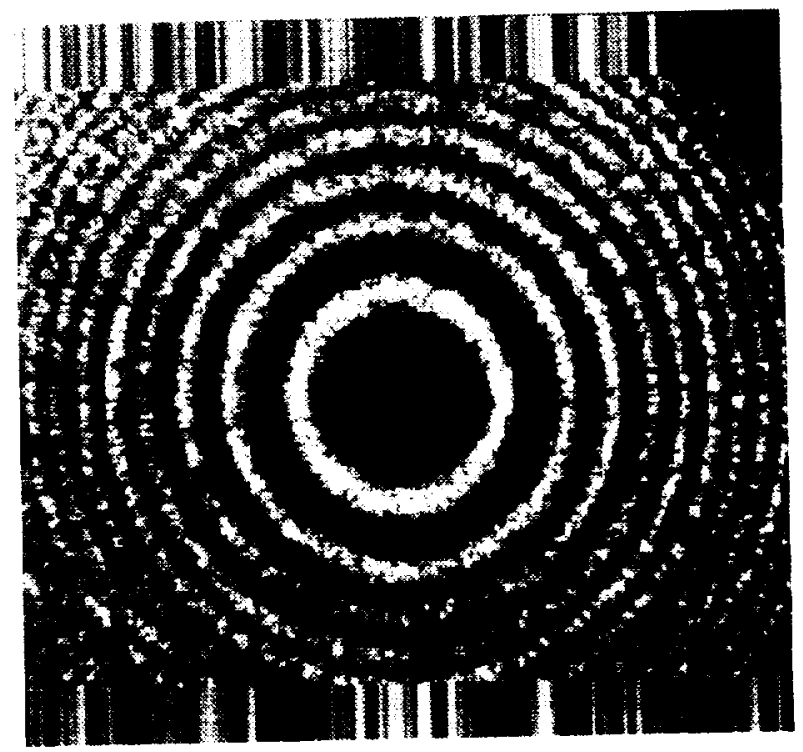

(d)

Fig, 2 Initial observations of the transmitted circular fringe pattern from a HeNe-illuminated FabryFig. 2 Inilal obsertern, (b) a dark count integration, (c) a flatPérot interferometer: (a) the original uncorrected suitably scaled fringe pattern after dark count, flat-field, field image of a white light source, and (d) the suitably scaled tringe patten aspect ratio corrections for the rectangular pixel size of the Fairchild CCD.

patterns were performed with the Fairchild CCD system. Figure 2 displays one of the first observations. Here a whitefrosted globe illuminated by the HeNe laser was used to scatter the laser radiation to evenly illuminate a 2 -in.-diam set of etalon plates. The interference pattern was then focused onto the Fairchild CCD by means of a simple lens. The image was digitized with an 8-bit frame grabber board, stored, and then displayed on a monitor attached to a Micro Vax II system. Figure 2(a) shows an original raw image, while a subsequent dark image is shown in Fig. 2(b). A flat-field image of a tungsten bulb illuminating the globe is shown in Fig. 2(c). These last two images are required for our image processing: the dark frame is removed from both the HeNe and the flat-field images, and then the dark-corrected HeNe image is divided by the dark-corrected flat field. The first technical problem that we encountered was caused by the rectangular size of the pixels within the Fairchild CCD. In addition, the CCD222 was developed with an interline transfer architecture in which adjacent vertical photosites are covered by opaque aluminum shields. After each integration, a charge is transferred from the exposed vertical columns to the adjacent shielded columns so that another integration may take place while the previous one is read. The opaque shield is $18 \times 18 \mu \mathrm{m}$. while the active photosites are $12 \times 18 \mu \mathrm{m}$. Hence, the initial images will never show a circular pattern when one is input, as observed. However, by applying an aspect ratio correction, a circular image may be formed as shown in Fig. 2(c). The utility of the Fairchild device, though, 
is limited by the opaque shields, because one-half of the fringe pattern will never be observed. This and the fact that the CCD222 quickly saturated with thermal noise made it unacceptable for airglow FPI observations.

The next set of test patterns was acquired with the Thomson-based CCD system. HeNe laser fringe profiles were of adequate quality and attempts were made to reduce radially the fringe pattern from a 3-D nature (two linear and one intensity dimensions) to the conventional intensity versus phase difference scheme. To measure geophysical parameters accurately from the resultant fringe profile requires a procedure to perform this reduction. ${ }^{9}$ We know that an ideal transmitted pattern from a FPI is represented by an Airy function that has intensity maxima at integral values of the order of interference $m$, given by

$m \lambda=2 \mu t \cos \theta$

where $\lambda$ is the wavelength of the incident radiation, $t$ is the gap between the etalon plates, $\theta$ is the angle of the light path through the etalon relative to the normal, and $\mu$ is the refractive index of the material or gas between the plates. The input angular dependence may be replaced by the relationship between radial distance from the center of the fringe pattern $r$ and the effective focal length of the system $f_{r}$.

$\theta=\tan ^{-1}\left(r / f_{0}\right)$

and because the angles are small, may be expanded in a Taylor series yielding

$\lambda=\frac{\mu t}{f_{n}^{2} m} r^{2}$.

Thus, the wavelength is linear with respect to the square of the linear distance from the center of a transmitted FPI fringe pattern.

Figure 3 displays a result from a simulation performed to test the sensitivity of the $r^{2}$ reduction technique to an accurate description for the center of the FPI pattern. Figure 3(a) shows the initial Airy profile as a function of radial distance using the appropriate instrumental values for the Thomson-based CCD system. The fringe profiles clearly bunch together as the phase difference term passes through several orders of interference. Here, the area covered by each physical pixel was further subdivided into 100 equal area subpixels. The effect of integrating the intensity levels of subminiature pixels onto actual size pixels is shown by Fig. 3(b). The hatched line indicates the original input Airy function, while the histogram display indicates what the CCD would actually observe. Applying the software aperture technique of Mulligan," a simulated $r^{2}$ version was generated and is shown in Fig. 3(c). A slight broadening occurs in the data reduction technique resulting in a reduction of peak intensity for different orders.

The next step in the simulation was to offset the center of the fringe pattern and to redo the $r^{2}$ reduction. Figure 4 (a) displays the simulated fringe pattern. Various runs were performed with a sample result shown in Fig. 4(b). Here the value of the center coordinate for one axis has been off set by one-half of a pixel dimension, resulting in a fringe profile indicated by the solid line. The dashed line shows the ex-

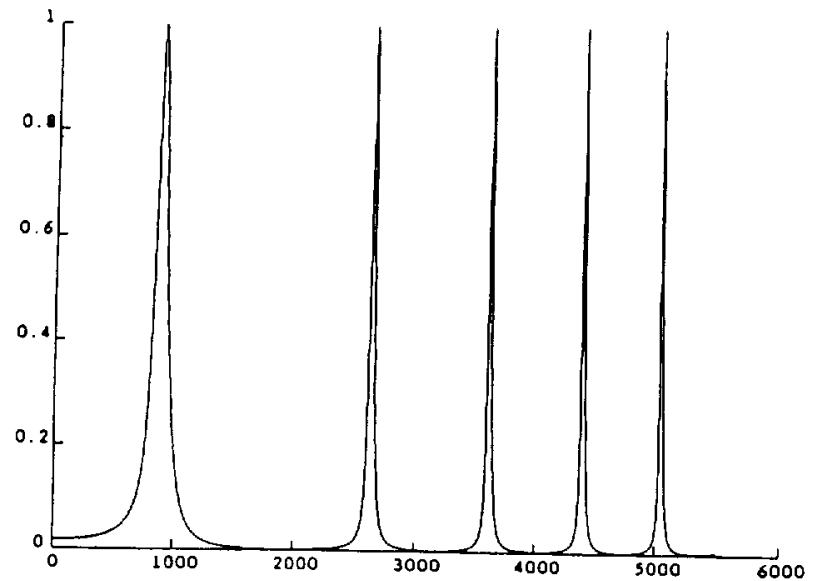

(a)

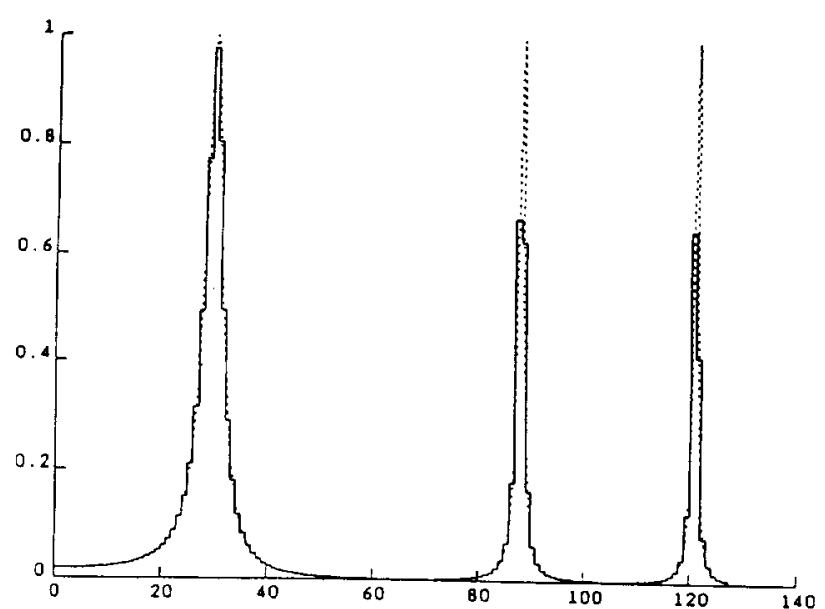

(b)

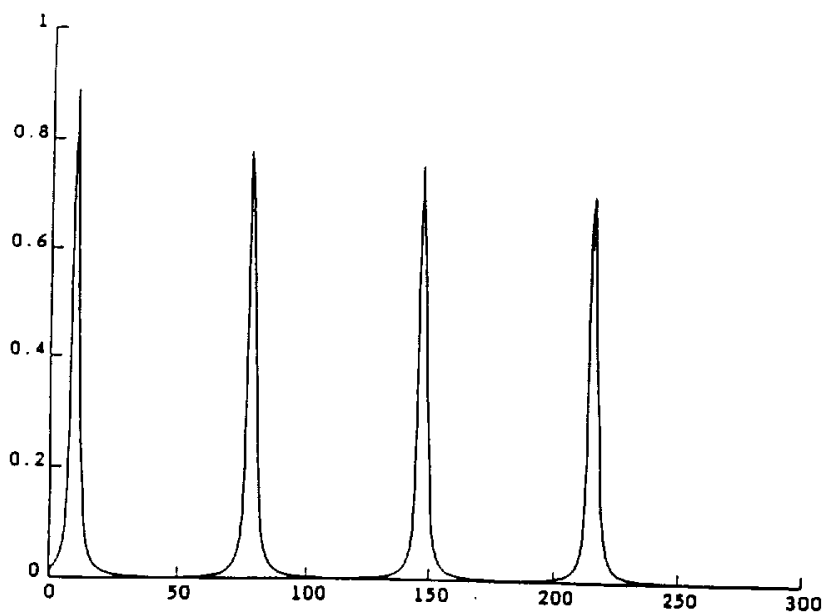

(c)

Fig. 3 (a) Airy profile simulation of a transmitted fringe pattern on illumination by a frequency-stabilized HeNe laser. Appropriate values for reflectivity, gap spacing, and the illuminating wavelength were included in the numerical simulation. The figure shows the expected input to a bare CCD. (b) The histogram function shows the simulated intensity pattern across a central axis of a bare CCD on illumination. The hatched line shows the input function. (c) The result of applying the $r^{2}$ reduction technique described in the text. The locations of maximum intensity are now evenly spaced as a function of bin number. 


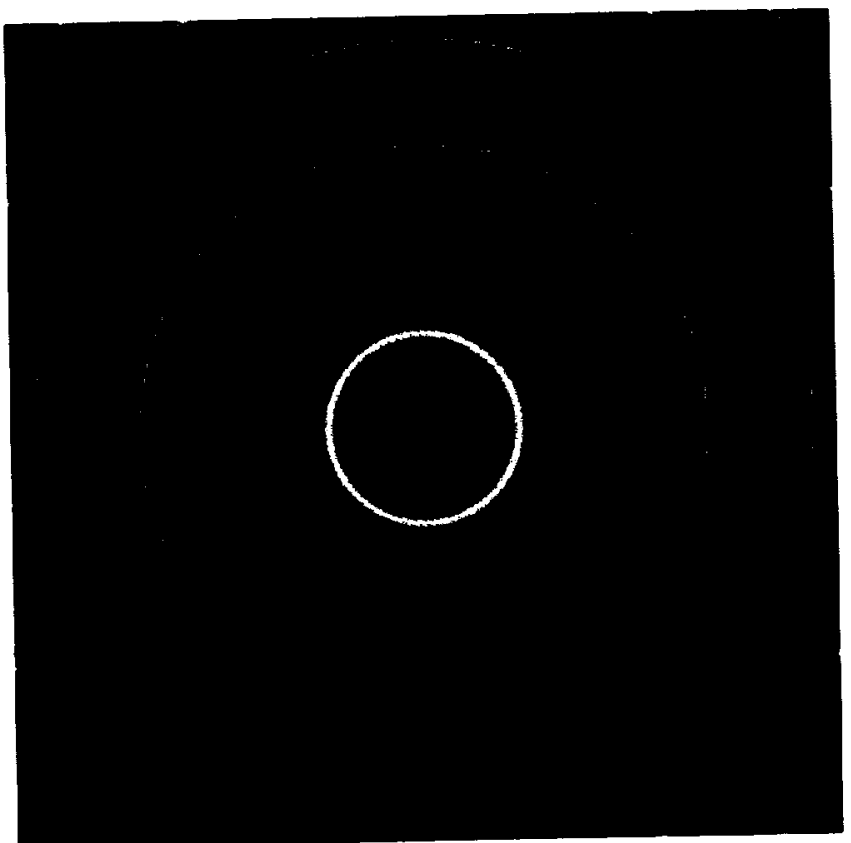

(a)

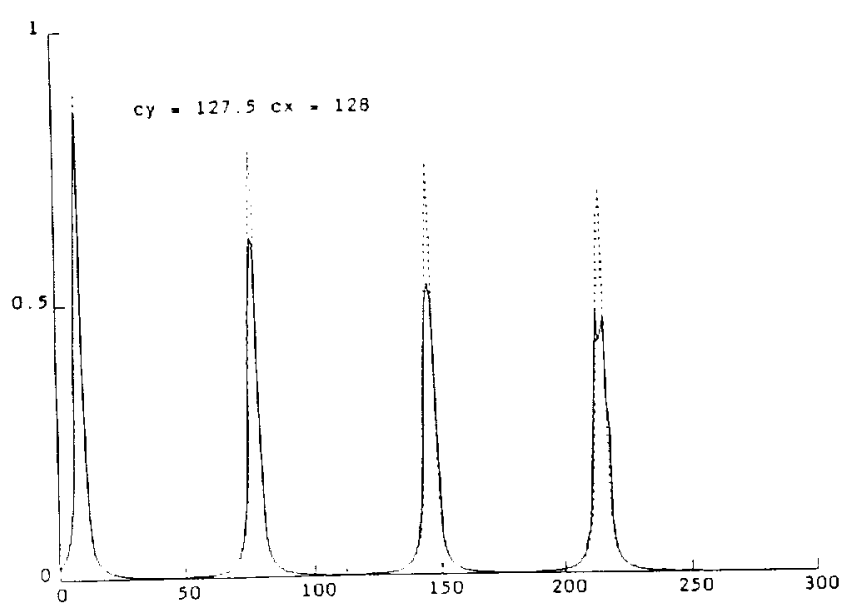

(b)

Fig. 4 (a) The input fringe pattern and (b) the result of mismatching the location of the center of (a). Here, the center was misplaced by one-half of 1 pixel, resulting in a broadened and doubled $r^{2}$ profile, compared to the anticipated profile shown as a dashed line.

pected profile for perfect alignment. Not only are the higher order fringes unnecessarily broadened, but a double peak appears because the simple $r^{2}$ reduction program cannot handle elliptical patterns. Typical HeNe finesse for the central fringe is of the order of 15 , but with poor alignment, outer fringes show tinesse values of 5 or less. Consequently, a great deal of energy is expended during start-up operations to ensure proper alignment of the optical elements for the best possible setup prior to remote unattended operation.

\subsection{Bias and Noise Reduction}

A difficulty encountered with low light level airglow observations is degradation caused by charge transfer efficiency
(CTE) problems, that is, losses due to inefficient transfer of charge from one photosite to the next as a CCD is read. Among various others, ${ }^{30}$ the Thomson TH7882 CDA sensor was known to exhibit a reduction in transfer efficiency when the number of electrons per pixel was small $\left(\sim 50 \mathrm{e}^{-}\right)$. The technique employed by Photometrics to improve the CTE was to illuminate the CCD weakly and uniformly with a preflash, or optical fat zero, equivalent to $100 \mathrm{e} /$ pixel, prior to any observation. As shown in Janesick et al., ") the CTE is dramatically improved when this technique is used. However, inherent in the addition of this bias charge to the electron count per pixel is an increase in the noise floor of the device due to the shot noise that the additional illumination provides. This noise is in addition to the other usual sources of noise: shot noise due to illumination by airglow or aurora, dark current generation due to charges of thermal origin, and preamplitier or readout noise. The normal techniques employed to improve the final image are to address the reduction of these noise sources by increasing the integration period to reduce the shot noise, to reduce the die temperature of the sensor to eliminate dark current generation, and to bin pixels on chip so that readout noise is only added to the "super pixel" rather than to the individual pixels.

Our observations of oxygen and hydroxyl airglow at midlatitudes, samples displayed in Fig. 1, are usually performed with a 5-min integration period. Initially, observations were performed such that the system gain was determined by the ratio of the full well capacity of each super pixel and the full range of the analog-to-digital converter. It was soon discovered that unnecessary digitization noise was being added to the final images - the difference between maximum intensity and minimum intensity values for airglow fringe profiles was of the order of unit values for typical profiles, in which the minimum value included the sky plus all noise components. Experiments were performed to optimize system gain for the greatest expected airglow signal values. In practical terms, this meant reducing the value for the analog-to-digital saturation limit to a much smaller number. This implied that the full well capacity was greatly underutilized and that shot noise components contained higher digital values. Histograms of 5-min dark count (plus bias) observations indicated that when a representative bias frame was removed from the dark frame, the average digital value was zero (ignoring random cosmic ray strikes and other "hot-pixel" values). Hence, dark count observations were no longer performed during sky observations to increase the observing frequency of airglow data, though dark frames may still be required for longer integrations.

The final sky frame still includes a contribution from the bias preflash. As shown in Janesick et al., "'CTE performance in a $C C D$ responds dramatically to die temperature. At the optimum system gain, the contribution of the bias component to the sky frame was still substantial and required a process to carefully estimate and remove it from the frane. A high SNR bias frame is usually acquired by averaging 81 separate bias frames prior to performing sky observations. The cryogenic camera heads that we use are temperature stabilized to within $\pm 0.1^{\circ} \mathrm{C}$, but at the system gains that are employed, we have found that average bias frame values slowly drift during long-term nighttime observations. This hehavior is associated with small temperature drifts immediately following the delivery of cryogen to the detector dewar, amounting 
to roughly $5 \%$ of the average nighttime bias value (note that the CCD is not operated at the cryogen temperature, but usually at $-100^{\circ} \mathrm{C}$ ). The routine used to remove the bias component from the sky frame utilizes this high SNR bias frame by subtracting this amount from the sky frame. We have found this to be adequate except during intervals when the dewar housing the camera head is being refilled with liquid nitrogen. Our operational answer to this problem was to control the refill timing to coincide with dusk and dawn, the latter to ensure that the detector dewar would remain cool during daylight periods. We note here that with the new Kodak KAF 1400 based system in Watson Lake, preflashing of the sensor is not used because airglow emissions are inherently brighter (due to the high latitude) and the etalon plates have a much larger area (6-in. diameter versus 4-in. diameter) resulting in higher count rates. The Watson Lake airglow sample shown in Fig. 1 (c) was acquired in $1 \mathrm{~min}$.

\subsection{Geophysical Observations}

Two stations are currently operating with bare CCD sensors: Watson Lake, Yukon, using the Kodak chip, and Peach Mountain, Michigan, using the Thomson device, though the PM512 chip has been thoroughly tested at the latter site. Figure 5 displays a sample from the first stage of processing the raw fringe data acquired in Watson Lake on day 60, 1992. Here the FPI was observing emission from the OI $(6300-\AA)$ feature in five different directions: vertical and at a 45-deg angle in the four cardinal directions. Both frames show the $r^{2}$ reduction results for the five measurements per cycle. The integration period per direction is determined by first acquiring a sample 10-s image. Watson Lake is sufficiently close to the northern auroral oval that the possibility of auroral contamination is quite high, and as such could saturate the image; hence, a quick integration is necessary per direction from which a time scaling factor is determined for the final recorded image. The range available is from the original 10 to $180 \mathrm{~s}$, determined primarily from a numerical simulation for anticipated uncertainty estimates for the line-of-sight winds and temperatures that may be extracted from the measurement; here, about $10 \mathrm{~m} / \mathrm{s}$ and $25 \mathrm{~K}$. respectively. The two sets of five fringe profiles clearly show the wavelength shifts that the bare CCD FPI combination is capable of measuringhere amounting to about $200 \mathrm{~m} / \mathrm{s}$ in the meridional direction.

The Peach Mountain station routinely observes emissions from the Meinel band of hydroxyl. This feature provides a means to measure the neutral winds in the mesosphere. Fringe profiles similar to those shown in Fig. 5 have been routinely acquired for the last several years. A data reduction technique has been developed derived from that described in Killeen and Hays. "Instrumental calibration is performed by pressure scanning the interferometer while observing a frequencystabilized HeNe laser. A set of 160 images is acquired providing a scan over two full free spectral ranges for each of the $r^{2}$ annular bins (in our analysis, we chose 150 such bins). The periodic behavior of the fringes is numerically reduced to Fourier coefficients, which are shifted in wavelength to the appropriate sky wavelength, here $8920 \AA$. Sky observations also contain instrumental contributions convolved with the actual Doppler-broadened skyline profile. The fitting procedure chosen is to assume a Gaussian profile for the airglow emission that contains four unknowns: (1) width of the profile
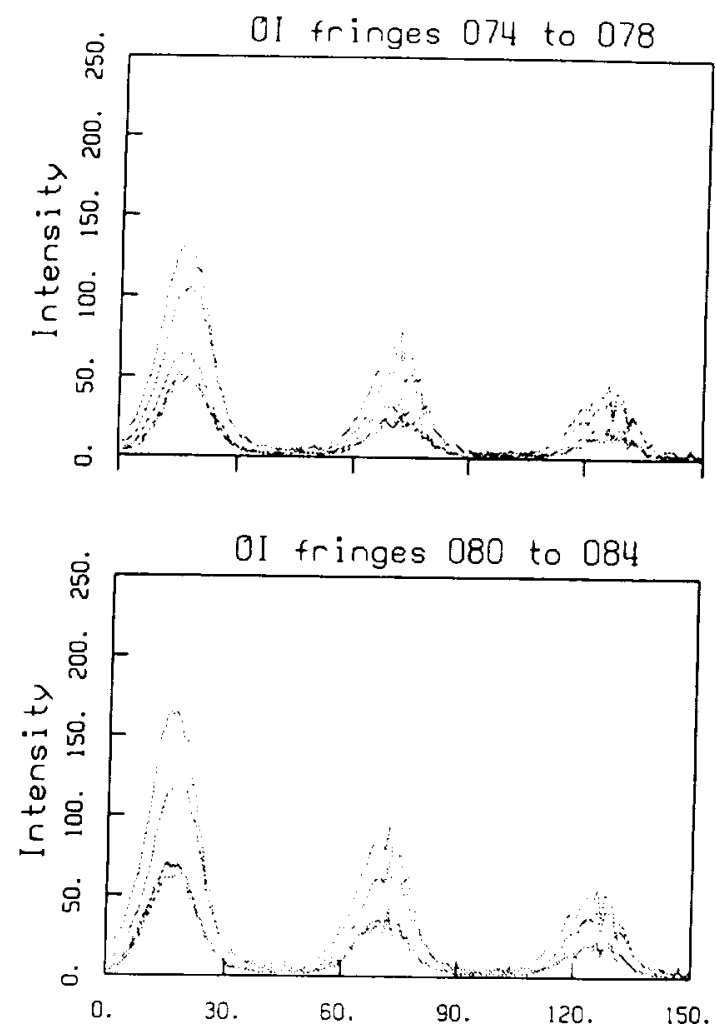

Fig. 5 Radially reduced OI (6300-A) airglow fringes acquired at Watson Lake, Yukon. Five fringes are shown in each panel. Shifts in peak location are evidence of strong horizontal neutral wind components.

that is proportional to the kinetic temperature of the emitting species, (2) wavelength shift of the center of gravity of the profile that is proportional to the line-of-sight Doppler velocity, (3) a scaling factor that is proportional to the absolute brightness of the emission feature, and (4) a remnant background that is proportional to weak sky continuum. This analytical representation of the airglow emission line profile is then convolved with the numerical representation of the instrumental profile at the appropriate wavelength and then compared with the actual observed $r^{2}$ profile. Better guesses, in a least squares sense, are then made for the four unknowns until convergence is reached. ${ }^{12}$ Figure 6 displays horizontal neutral wind results for two successive periods ${ }^{13}$ in 1990. The meridional (N,S) and the zonal (E, W) components are shown with error bars as a function of local time, with $N$ and $\mathrm{E}$ being positive. Both low- and high-frequency temporal variations are evident in the wind component data, and these are current topics of study within the aeronomical community.

\section{Conclusions}

The current set of scientific grade CCDs has been shown to be capable of performing aeronomical quality observations of airglow emissions with Fabry-Pérot interferometers. Both routine (OI 6300- $\AA$ ) and unique (Meinel OH 7.3 band) observations have been acquired with several different bare CCDs. The fringe quality is satisfactory and standard reduction techniques are able to extract high-quality geophysical data from the observations. The CCD is a versatile sensor 

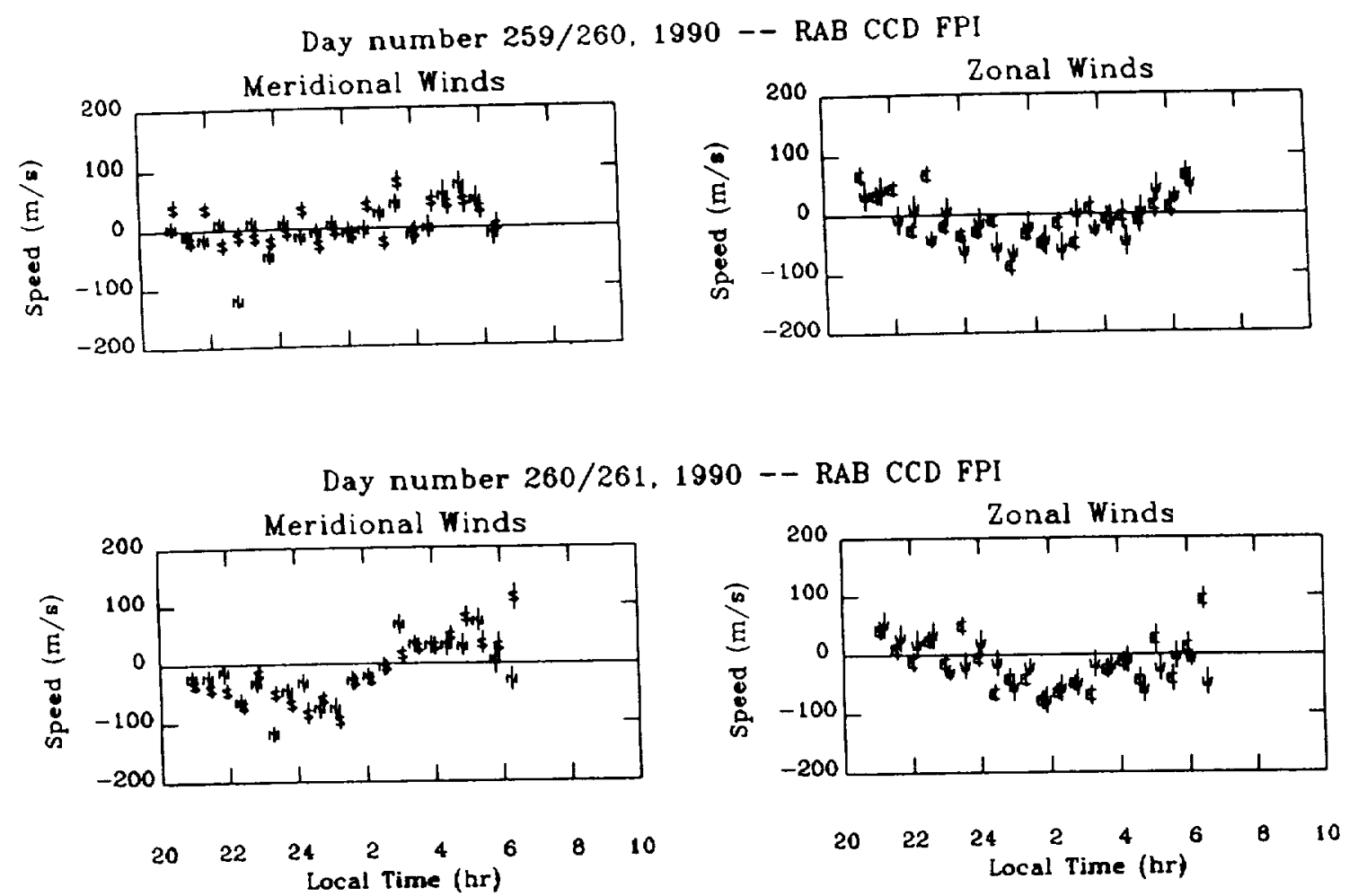

Fig. 6 Wind determination from fringe profiles of the Meinel $(7,3)$ band of hydroxyl as observed at a Fig. 6 Wind determination from fringe profles of the morizontal neutral wind data are displayed as meridional mid-latitude site. Two successive nights of horizontal neutral wind data are displayed as mertical bar indicating and zonal components. The character refers to the dired

and, with proper precautions, may be used in an unmanned automatic data-taking mode with little or no maintenance.

Several initial problems have been addressed and solutions have been found: (1) the use of full-frame transfer devices with square pixels is far superior to the interline transfer devices, (2) cooling of the sensor and the use of the MPP mode are satisfactory for the integrations required for the brighter airglow emission features, (3) careful attention to the choice for the center of a circular fringe pattern is necessary to reduce unnecessary broadening of the $r^{2}$ fringe profile and to keep the useful finesse as high as possible, (4) chips that require an optical fat zero or bias contribution must be carefully characterized for low light level observations, and (5) standard data reduction techniques may be easily applied to data acquired by bare CCD-based FPls.

The bare CCD has quickly revolutionized the method of performing unattended remote field site observations of the thermodynamics of atmospheric regions. The detector is robust and has an excellent quantum efficiency over the spectral regions of interest, a satisfactory dynamic range, and a linearity in response at the count rates acquired. Our group is planning to complete the upgrade of the set of field sites that we maintain and is currently considering further novel modifications to lab-based CCD FPI systems, such as imaging fringes from two different frequencies simultaneously on the same device and imaging fringes onto concentric ring CCDs.

\section{Acknowledgments}

We would like to express our appreciation to the Space Physics Research Laboratory, which provided some of the initial start-up funding for our bare CCD program. Dr. F. G. McCormac (Queens University, Belfast) was responsible for much of the initial work with the Thomson-based CCD system. We acknowledge the contributions of Dr. F. J. Mulligan (St. Patrick"s College, Maynooth, Eire) during his summer visits to Michigan. The contributions of many students, funded by NSF grant ATM-8804896, are acknowledged, in particular, Darryl Barlett, Michael Brennan, Catherine Rivers, Gerard Roe, and Lisa Gillikin. Dr. Niciejewski, Dr. Killeen, and Matthew Turnbull were funded by NSF grants ATM-8822530, ATM-9002607, and ATM-9002608. Grateful assistance by the Astronomy Department is acknowledged, in particular, Prof. D. Richstone for the initiation of our presence at Peach Mountain and George Latimer for his constant vigilance at the site. The Watson Lake, Yukon, operation could not have succeeded without the kind assistance of Peter Skerget and Tom Marche of Northwestel. We sadly note the passing of Michael Caspersen, who played an important role in the construction and initiation of the Watson Lake station.

\section{References}

1. G. Hernandez, Fabri-Péran Interferometers, Cambridge University Press Cambridge (1986)

2. F. Jacka, "Application of Fabry-Pérot spectrometers for meatsuremen of upper atmosphere temperatures and winds." MAP Handbesk 13 R A Vincent. Ed.,pp. 19-40, SCOSTEP. University of Illinois (1984)

R. A. Killeen. B. C. Kennedy, P. B. Hays, D. A. Symanow, and D. H . L. Killeen. B. C. Kennedye dector for the Dynamics Explorer FabryCeckowski, "Image plane detector for the Dynamics Explor

J. W Chumberlain. Phrsics of the Aurora and Airglow. pp. 564-571. Academic Press, New York 1961$).$ 
5. J. W. Meriwether, Jr.. C. A. Tepley, S. A. Price, P. B. Hays, and L. L. Cogger, "Remote ground-based observations of terrestrial airglow emissions and thermospheric dynamics at Calgary. Alberta. Canada," Opt. Eng. 22(1), 128-131 (1983)

6. M. R. Torr, D. G. Torr, R. Baum, and R. Spielmaker. "Intensified-CCD focal plane detector for space applications: a second generation." $\mathrm{Appl}$. Opt. 25, 2768-2777 (1986).

7. E. S. Trudell, T. L. Killeen, R. J. Niciejewski, Su. Basu, and B. W Reinisch. "An investigation of a polar cap arc sequence using ground based measurements at Thule, Greenland," EOS Trans. Am. Gerohn. Union 71, $581(1990)$

8. J. Janesick, T. Elliott, R. Bredthater, C. Chandler, and B. Burke, "Fanonoise-limited CCDs," Proc. SPIE 982, 70-95 (1988).

9. F. J. Mulligan. "A new technique for the real-time recovery of FabryPérot line protiles," J. Phys. E: Scientific Instrum. 19, 545-551 119861.

10. J. R. Janesick. T. Elliott, S. Collins, M. M. Blouke, and J. Freman, "Scientific charge-coupled devices." Opt. Eng. 26(8), 692-714 (1987)

11. T. L. Killeen and P. B. Hays, "Doppler line protile analysis for a mullichannel Fabry-Pérot interferometer," Appl. Opt. 23, 612-620 (1984).

12. P. R. Bevington. Data Redaction and Error Analysis for the Phrsica Sriences, pp. 232-245, McGrau-Hill. New York (1969)

13. M. Turnbull, T. L. Killeen, R. J. Niciejewski. and G. S. N. Murly, "Mesospheric wind measurements based on observations of the hydroxyl radical using a bare CCD," EOS Trans. Am. Geophss. Union 71, 1486 $(1990)$.

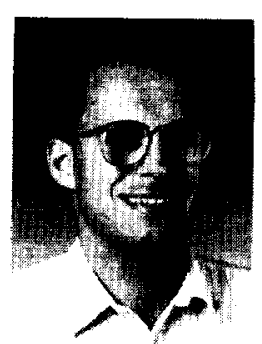

Rick Niciejewski received an MSc in 1981 and $\mathrm{a} P \mathrm{PhD}$ in 1987 from the University of Western Ontario in London, Canada. He joined the Space Physics Research Laboratory of the Atmospheric, Oceanic, and Space Science Department of the University of Michigan as a postdoctoral research fellow in 1987 and became an assistant research scientist in 1990. His current research interests are in the field of experimental aeronomy including optical observations of the terrestrial airglow and aurora. Primary topics of interest are the thermodynamics and weather of the terrestrial upper atmosphere and the application of state-of-the-art technology to remote diagnostics.

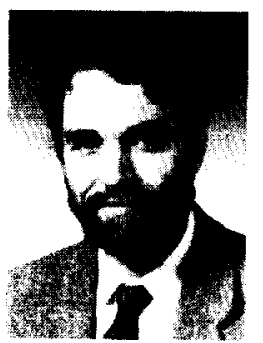

Timothy L. Killeen is a professor of atmospheric and space sciences at the University of Michigan. He received a BSc (first-class honors) in physics in 1972 and a $\mathrm{PhD}$ in atomic and molecular physics from University College London in 1975. Dr. Killeen is the director of the Space Physics Research Laboratory and leads a research group of 15 scientists and engineers working in the field of aeronomy. His research involves using a combination of theoretical and experimental techniques to investigate the dynamics, chemistry, and composition of the upper atmospheres of the Earth and other planets. Dr. Killeen has authored or coauthored more than 100 publications in refereed journals. He is the principal investigator for more than 10 ongoing research projects for NASA, NSF, and the U.S. Air Force. He is also the principal investigator for a chain of ground-based optical observatories stretching from Northern Greenland to Michigan.

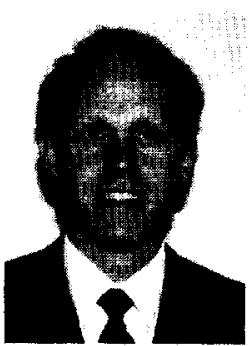

Matthew Turnbull received an MSc degree in aeronomy in 1992 from the Space Physics Research Laboratory at the University of Michigan. His primary research topics included Fabry-Pérot interferometer observations of the terrestrial airglow from middle and Arctic latitudes as well as allsky imagery of auroral motions. 


